\title{
Bilateral Coronary Ostial Stenosis after Bentall Procedure in a Patient with Marfan Syndrome
}

\author{
Milán Vecsey-Nagy ${ }^{1}$, Ádám Jermendy ${ }^{1}$, György Szabó ${ }^{2}$, Kálmán Benke ${ }^{1}$, Zoltán Szabolcs ${ }^{1}$, \\ Béla Merkely ${ }^{1}$, Pál Maurovich-Horvat ${ }^{1}$ \\ ${ }^{1}$ MTA-SE Cardiovascular Imaging Research Group, Heart and Vascular Center, Budapest, Hungary \\ ${ }^{2}$ Uzsoki Cardiovascular Center, Budapest, Hungary
}

\section{ABSTRACT}

Introduction: The majority of patients with Marfan syndrome undergo Bentall procedure. Several periprocedural complications may arise; cardiac complications play the most dominant role. Case presentation: A patient with Marfan syndrome developed symptoms two years after the Bentall procedure. Coronary СT angiography revealed bilateral coronary ostial stenosis. Percutaneous coronary intervention was performed, and two drug-eluting stents were implanted. Conclusions: Coronary CT angiography is a valuable tool for the examination of symptomatic patients following surgical aortic root replacement.

Keywords: coronary CTA, percutaneous coronary intervention, Bentall procedure

\section{ARTICLE HISTORY}

Received: November 1, 2017

Accepted: November 15, 2017

\section{CORRESPONDENCE}

Pál Maurovich-Horvat

Határőr u. 18, H-1122 Budapest, Hungary Tel: +3620 6632485

E-mail: p.maurovich.horvat@ mail.harvard.edu.

\section{INTRODUCTION}

Marfan syndrome is an autosomal dominant disease of the connective tissue. Its cardiovascular manifestation plays a major role in the mortality of patients with Marfan syndrome. Cardiovascular manifestations of the disease include mitral valve prolapse, insufficiency of the aortic and mitral valves, and dilatation of the aortic root and the ascending aorta.

Severe dilatation of the aortic root requires reconstructive surgery, for which the most widespread technique is the Bentall procedure. The concept of this surgical technique is to replace the dilated aortic root with an artificial vessel conduit and a prosthetic aortic valve. The orifices of the coronary arteries are reimplanted to the conduit.

\section{CASE PRESENTATION}

We present a case of a 24-year-old female patient with Marfan disease, who underwent Bentall procedure due to a rapidly progressing aortic root dilatation and grade III aortic valve insufficiency. The patient developed a thirddegree atrioventricular block with junctional rhythm perioperatively, which resolved during her hospital stay. A second-degree atrioventricular block with a tendency 

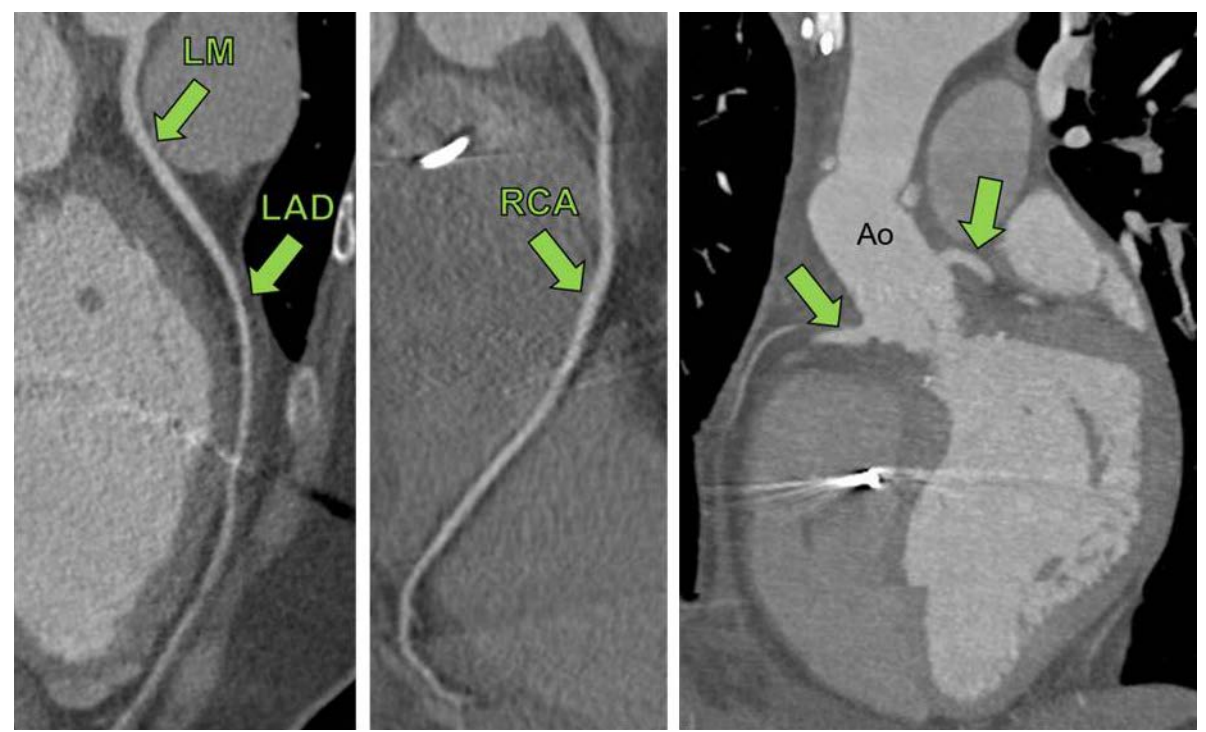

FIGURE 1. Coronary CTA six weeks after the Bentall procedure. The orifices of the reimplanted left main artery (LM) and the right coronary artery (RCA) are normal. Green arrows indicate the coronary arteries and the orifices of the coronaries.

of bradycardia remained in the following weeks, without the indication for permanent pacemaker implantation. Six weeks after the Bentall procedure, ventricular tachycardia was detected, followed by ventricular fibrillation. After a short cardiopulmonary resuscitation, the patient was stabilized, and an implantable cardioverter defibrillator (ICD) was implanted. In order to assess the orifices of the reimplanted coronary arteries, coronary computed tomography angiography (CTA) was performed. No stenosis was visible in the orifices of the left main and the right coronary arteries (Figure 1). The patient was discharged from the hospital.
Two years later, follow-up coronary CTA was performed due to atypical chest pain, exercise-induced shoulder pain, nausea, and multiple adequate ICD shocks. On the coronary CTA images, critical pre-occlusive orificial stenoses of both the left main and right coronary arteries were visualized (Figure 2).

Urgent invasive coronary angiography was performed, which confirmed the findings of the coronary CTA (Figure 3).

Percutaneous coronary intervention was performed on both vessels, with the implantation of two drug-eluting stents in the LM and RCA orifices (Figure 4.)
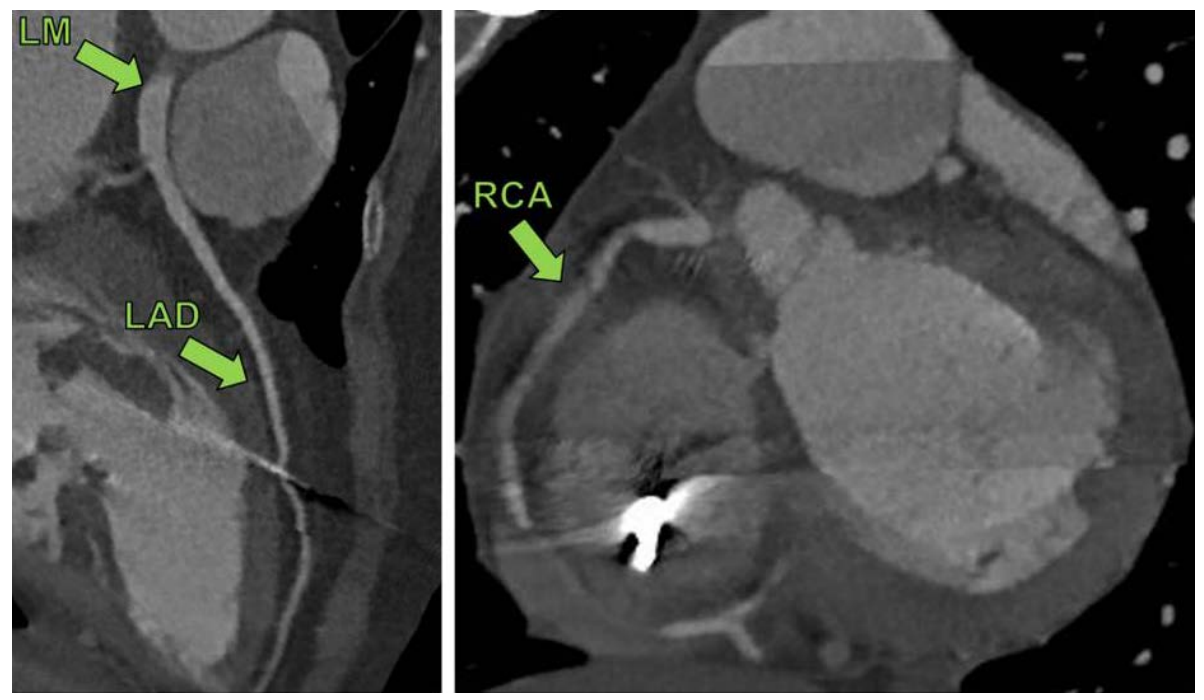

FIGURE 2. Pre-occlusive stenoses were visualized in the orifice of the left main and right coronary artery (arrow heads) 

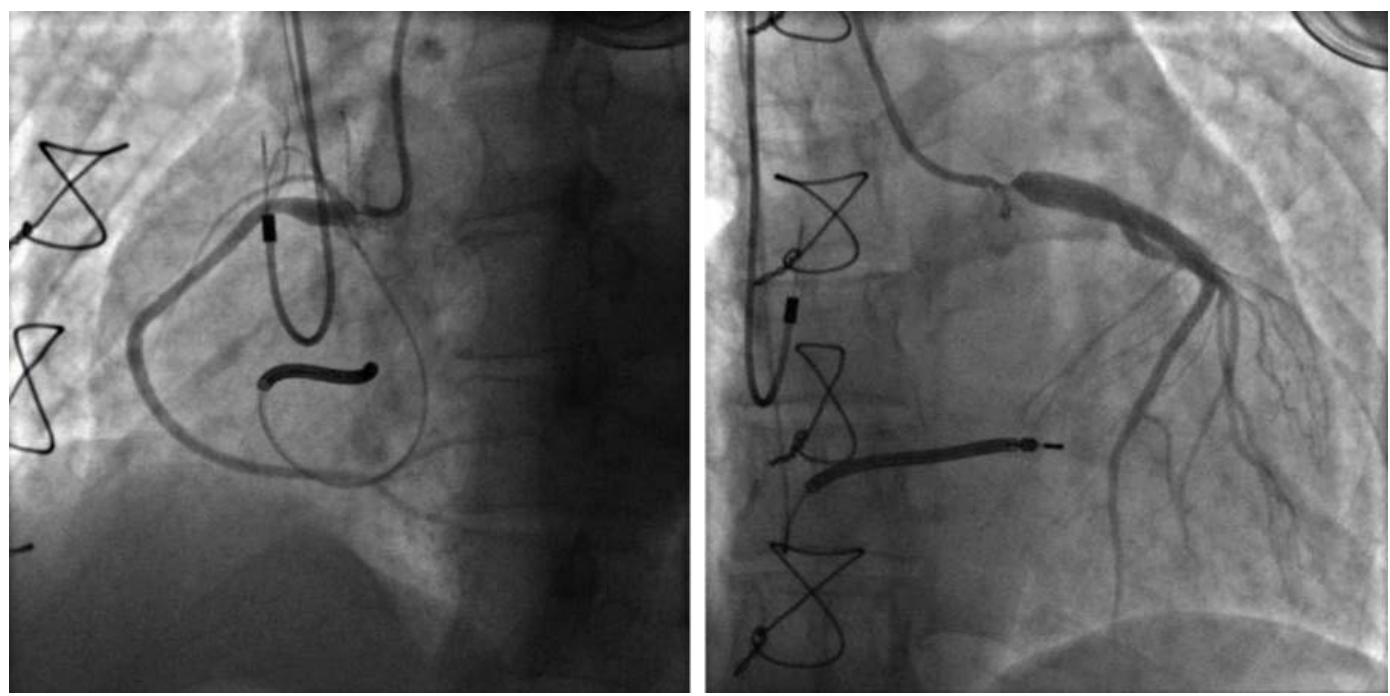

FIGURE 3. Pre-occlusive stenoses in the orifice of the RCA and LM (arrow heads)

Six months after the intervention, a follow-up coronary CTA was performed. The implanted stents were visualized in a normal position, and in-stent restenosis was ruled out (Figure 5).

The patient agreed to the publication of his data, and the institution where the patient had been admitted approved the publication of the case.

\section{DISCUSSION}

Although the most apparent clinical manifestations of Marfan syndrome are those regarding the skeletal system, when considering overall mortality, the involvement of the cardiovascular system is the most dominant factor. ${ }^{1}$ In recent years, cardiovascular imaging modalities (echocar- diography, cardiac CT, cardiac MRI) have become inevitable tools in screening, diagnosing, and life-long monitoring of the disease. A pathologic dilatation of the aortic root is characteristic to the Marfan population, being present in approximately $75 \%$ to $85 \%$ of the patients. ${ }^{2}$ Since its introduction in 1968, the Bentall procedure has been considered the treatment of choice in patients requiring aortic root replacement. According to recent literature, prophylactic aortic root replacement is a recommended method to improve the prognosis of patients with Marfan syndrome. ${ }^{3}$

Iatrogenic coronary ostial stenosis has been described as a potential result of procedures involving aortic valve replacement. ${ }^{4}$ Stenoses of any etiology in the ostia of the coronaries following Bentall procedure can result in various cardiac-related short- and long-term adverse
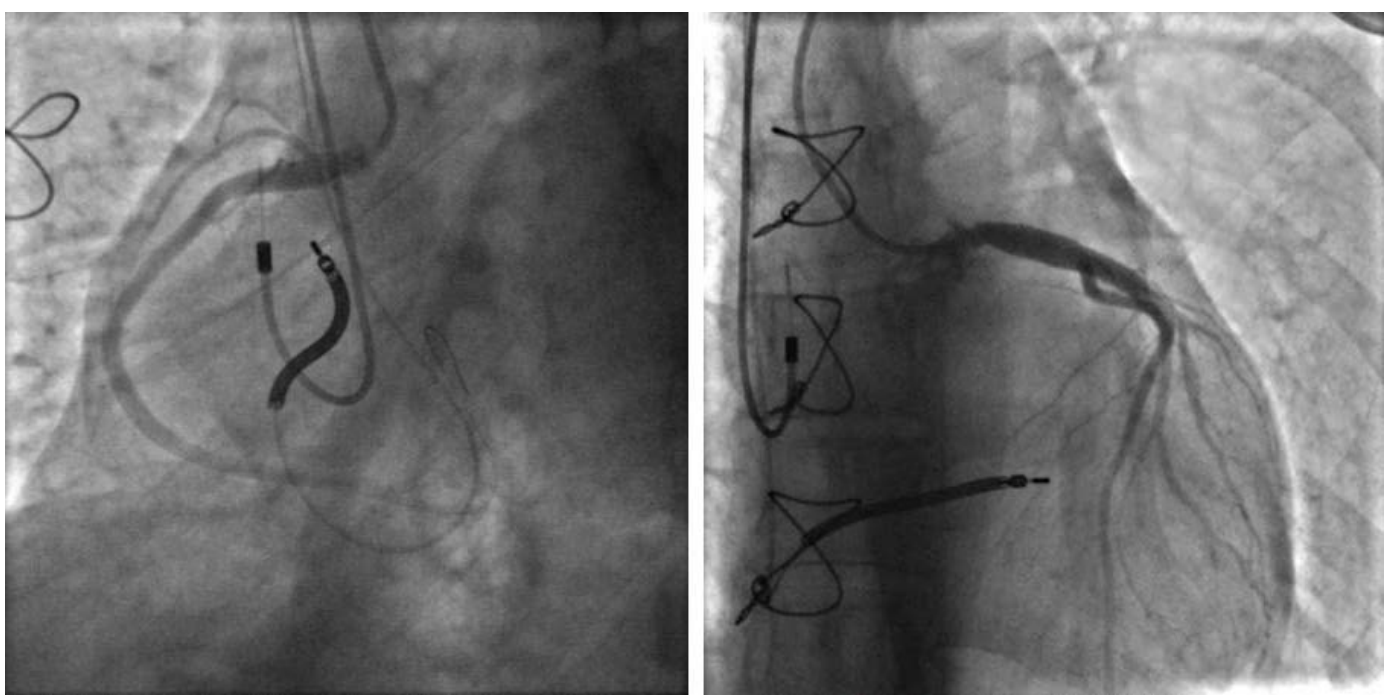

FIGURE 4. RCA and LM following PCI and stent implantation 

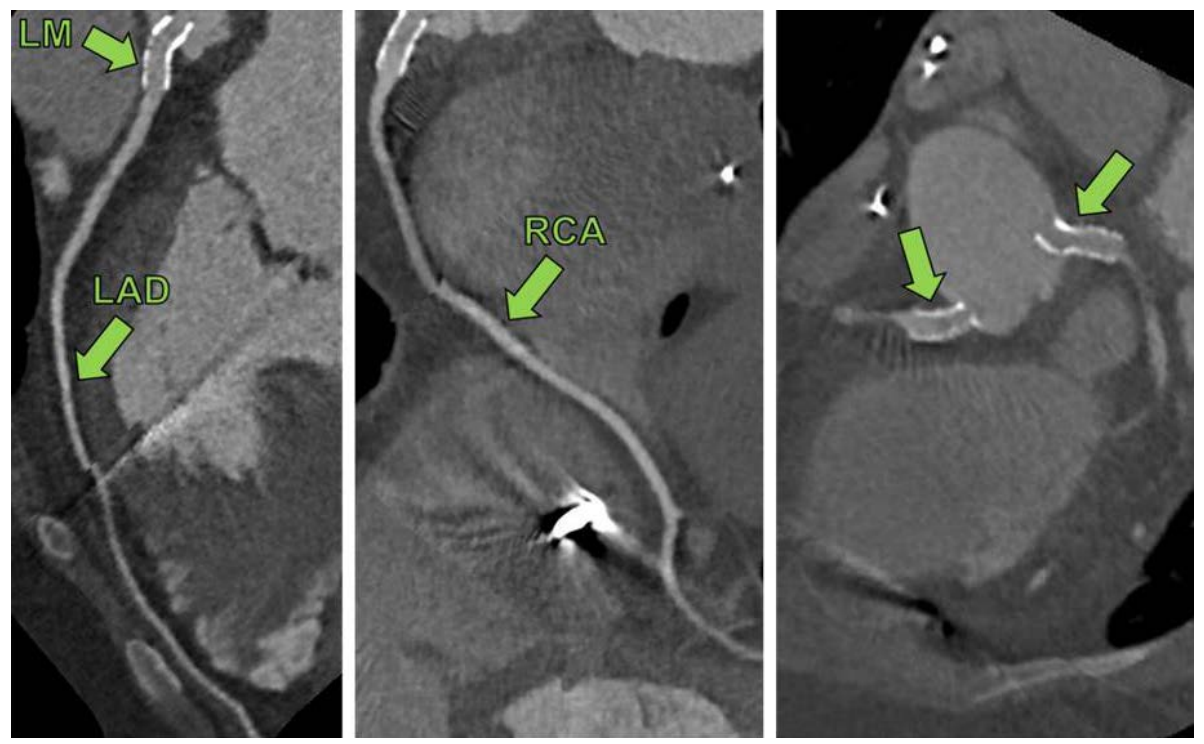

FIGURE 5. Coronary CTA six months after the $\mathrm{PCl}$ showed intact stents in both the orifice of the RCA and LM (arrows)

outcomes. These include low cardiac output syndrome, ventricular arrhythmias, congestive heart failure, and myocardial infarction. ${ }^{5}$

\section{CONCLUSION}

It is important to note that complications affecting the coronary arteries after Bentall procedure are rare, and they are associated with high mortality. Coronary CTA allows precise assessment of the orifices of the reimplanted coronary arteries, thus making it a useful diagnostic tool for the examination of symptomatic patients following surgical aortic root reconstruction.

\section{CONFLICT OF INTEREST}

None declared.

\section{ABBREVIATIONS}

CPR cardiopulmonary resuscitation

CCTA coronary computed tomography angiography
DES drug-eluting stent

ICD implantable cardioverter defibrillator

LM left main coronary artery

MRI magnetic resonance imaging

PCI percutaneous coronary intervention

RCA right coronary artery

\section{REFERENCES}

1. Stuart AG, Williams A. Marfan's syndrome and the heart. Arch Dis Child. 2007;92:351-356. doi: 10.1136/adc.2006.097469.

2. Sheick-Yousif B, Sheinfield A, Tager S, et al. Aortic root surgery in Marfan syndrome. Isr Med Assoc J. 2008;10:189193.

3. Benke K, Ágg B, Szabó L, et al. Bentall procedure: quarter century of clinical experiences of a single surgeon. J Cardiothorac Surg. 2016;11:19. doi: 10.1186/s13019-0160418-y.

4. Bernelli C, Bezante GP, Brunelli C, Balbi M. Iatrogenic left main coronary ostial stenosis: after a Bentall procedure in an asymptomatic young man. Tex Heart Inst J. 2012;39:393397.

5. Joo HC, Chang BC, Yoo KJ, Lee S. Clinical experience with the Bentall procedure: 28 years. Yonsei Med J. 2012;53:915-923. doi: 10.3349/ymj.2012.53.5.915. 